\title{
CRACK FORMATION AND CRACK PROPAGATION INTO THE COMPRESSION ZONE ON REINFORCED CONCRETE BEAM STRUCTURES
}

\author{
SAMDAR KAKAY, DANIEL BÅRDSEN \& OVE T. GUDMESTAD \\ Department of Mechanical and Structural Engineering and Material Science, University of Stavanger, Norway.
}

\begin{abstract}
Crack formations in concrete may cause major damages in concrete structures. These damages require extensive maintenance work and thus have high costs. This paper addresses issues such as what causes cracks in concrete structures and how does the appearance of cracks look like with respect to an applied load? Can the appearance, distance, and size of the crack tell us something about crack initiation and propagation, or is it just by pure coincidence that cracks occur in structures as they do?

This research work investigated the effect of external factors such as load variables, time, the dimensions of the beam and the relative humidity on crack formation. Internal factors that have been investigated are the various constituents of the concrete, and how various levels of these constituents have an impact on cracking. In addition, the influence of concrete quality, tensile reinforcement, shear reinforcement, and anchoring reinforcement was investigated.

The paper presents technical calculations, where both the bending moment and shear forces are included in the analysis to determine how crack formations will propagate in the beam as a function of the applied loads. The first part of the paper deals with the theoretical factors that influence cracking in concrete. The second part deals with the calculations of crack formation in concrete. The results show how the cracks propagate in the $\mathrm{x}$ and $\mathrm{y}$ directions as a function of the load being applied.

Keywords: crack formation in the compression zone, crack propagation in $x, y$ axes.
\end{abstract}

\section{INTRODUCTION}

Since the behavior of cracks in the compression zone of a concrete beam is not well documented in literature, the primary objective of this paper is to study how cracks are formed and the behavior of their propagation into the compression zone. The method is based on the fact that a crack will be formed when $\sigma_{1}$ exceeds the characteristic tensile strength of the concrete (on the tensile loaded side of the beam).

This research paper deals with the theoretical and the technical aspects of how and why crack formation in concrete structures occurs. The technical part deals with fundamental classical mechanics, which describe the mathematical formulation of how cracks are formed in a supported beam element.

All the calculation examples are based on a simply supported concrete beam with dimensions $250 \times 300 \times 2200 \mathrm{~mm}$ and with 2 bars $\varnothing 12 \mathrm{~mm}$ in the compression zone and 3 bars $\varnothing 20 \mathrm{~mm}$ in the tension zone, as shown in Fig. 1. The beam is dimensioned for the ultimate failure load limits, namely: $90 \mathrm{kN}$ shear force and a bending moment of $63 \mathrm{kNm}$. The beam elements have been designed and tested in the laboratory at the University of Stavanger. From the study, a reasonable agreement between the theoretical calculation and the measured practical results are documented, see also Section 3, which presents the theoretical description of how crack propagation into the compression zone occurs.

Initially, a theoretical analysis on how wide the crack would be and to calculate the crack distance, as well as describing theoretically how the crack looks like was carried out, [1]. 
However, an important parameter, which controls the strength/resistance of the concrete against crack formation, is the water/cement ratio. Technical literature studies and calculations are performed according to Eurocode 2, Design of concrete structures, [2, 3].

\section{THEORETICAL CALCULATION OF CRACK FORMATION IN A STATICALLY LOADED REINFORCED CONCRETE BEAM}

From a theoretical point of view, it is well known that crack formation is caused by externally applied loads. However, there are also several other factors that influence the formation of cracks in concrete.

Cracks will be formed when $\sigma_{1}=\mathrm{f}_{\text {ctk } 0.05}$ where: $\sigma_{1}=$ principal stress, $\mathrm{f}_{\text {ctk } 0.05}=$ concrete characteristic tensile strength.

The state of stress on element 1 of Figs. 1 and 2 is given as:

$$
\sigma=\left[\begin{array}{ll}
\sigma_{x} & \tau_{x y} \\
\tau_{x y} & \sigma_{y}
\end{array}\right]=\left[\begin{array}{cc}
\sigma_{x} & 0 \\
0 & 0
\end{array}\right]
$$

$\sigma_{x} \neq 0$ (Assuming that the strain is sufficiently low for the concrete to take tension under the neutral axis).

We considered the principal stress given in eqn (1): The Principal stress is calculated from the normal and shear stresses in the x-y plane:

$$
\begin{gathered}
\sigma_{1,2}=\frac{\sigma_{x}+\sigma_{y}}{2} \pm \sqrt{\left(\frac{\sigma_{x}-\sigma_{y}}{2}\right)^{2}+\tau_{x y}^{2}} \\
\sigma_{1,2}=\frac{\sigma_{x}}{2} \pm \sqrt{\frac{\sigma_{x}^{2}}{4}} \quad \text { Whereas } \sigma_{1}=\sigma_{x}, \sigma_{2}=0 .
\end{gathered}
$$

Equation (2) gives the crack-propagation angle.

The crack angle for element 1 will be:

$$
\tan (2 \theta)=\frac{2 \tau_{x y}}{\sigma_{x}-\sigma_{y}} \quad 2 \theta=0,2 \theta=180=>\theta=90^{\circ}
$$

Considering element 2, the state of stress is given as:

$$
\sigma=\left[\begin{array}{cc}
\sigma_{x} & \tau_{x y} \\
\tau_{x y} & \sigma_{y}
\end{array}\right]=\left[\begin{array}{cc}
0 & \tau_{x y} \\
\tau_{x y} & 0
\end{array}\right]
$$

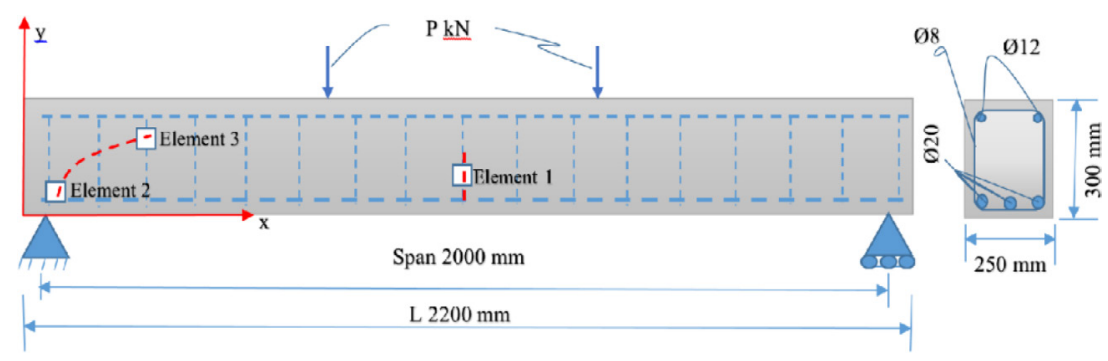

Figure 1: Simply supported reinforced concrete point loaded beam cross section. 


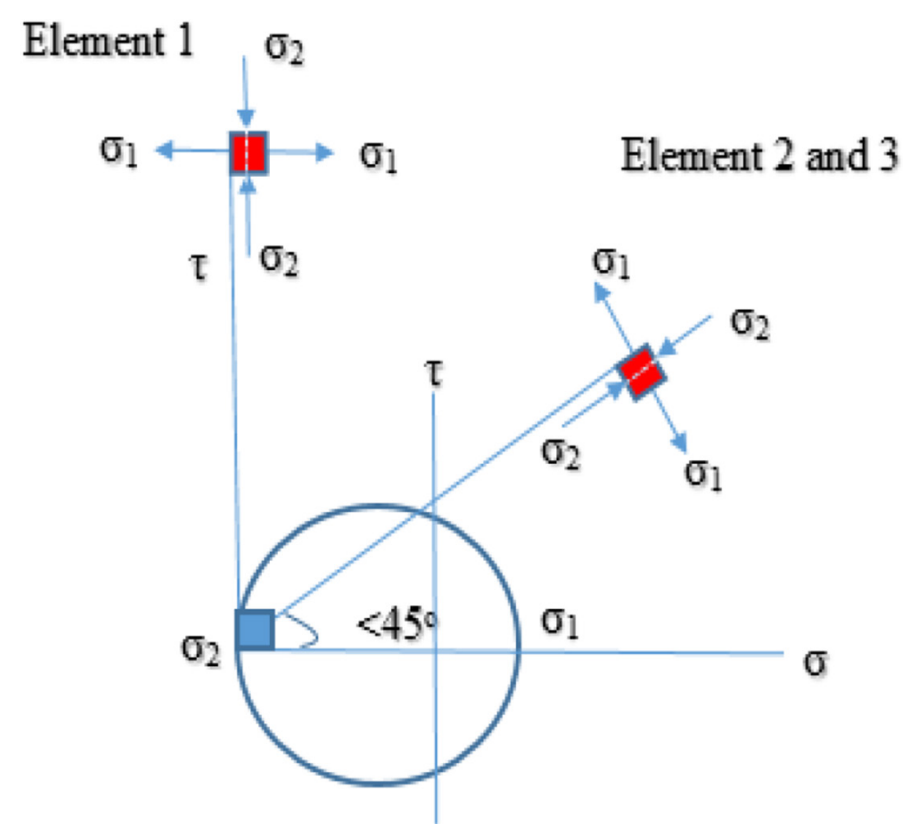

Figure 2: Stress distribution for elements 1, 2 and 3.

The principal stresses will be: $\sigma_{1,2}= \pm \tau_{x y}$, If we imagine that $\left(\sigma_{x}-\sigma_{y}\right)$ is infinitely small, then $\tan (2 \theta)=\infty$ and the crack angle will be $2 \theta=90^{\circ}=>\theta=45^{\circ}$

Considering element 3 : the state of stresses and the principal stresses are given as:

$$
\sigma=\left[\begin{array}{ll}
\sigma_{x} & \tau_{x y} \\
\tau_{x y} & \sigma_{y}
\end{array}\right]=\left[\begin{array}{cc}
\sigma_{x} & \tau_{x y} \\
\tau_{x y} & 0
\end{array}\right]=>\sigma_{1,2}=\frac{\sigma_{x}}{2} \pm \sqrt{\frac{\sigma_{x}^{2}}{4}+\tau_{x y}^{2}}
$$

Since element 3 is located in the compression zone, the normal stress along the $\mathrm{x}$-direction is $\sigma_{x}$ and by sign convention, the stress will have a negative value. We assume that $\tau_{\mathrm{xy}}$ has a large enough value compared to $\sigma_{x}$. This implies according to eqn (2) that:

$$
\tan (2 \theta)=\frac{2 \tau_{x y}}{\sigma_{x}-\sigma_{y}}=>2 \theta<\tan ^{-1}(\infty)=>2 \theta<90^{\circ}=>\theta<45^{\circ}
$$

\section{ELEMENT MODELLING AND CRACK PROPAGATION OVER NEUTRAL AXIS}

The method for estimating crack propagation is based on simple beam theory. Since the concrete is not homogeneous, the strength varies from place to place within the beam element. A crack will be formed at the location where crack initiation requires the least amount of energy. This method does not estimate how the crack will start such as FEM software's are capable of. However, we could use the formula to estimate the crack propagation angle when we know where the crack is formed. Figure 3 shows the comparison between the calculated and the experimental observation of the crack propagation.

\section{Modelling of Element 1:}

We look at eqn (3), which is derived from the internal moment of resistance of the beam. 

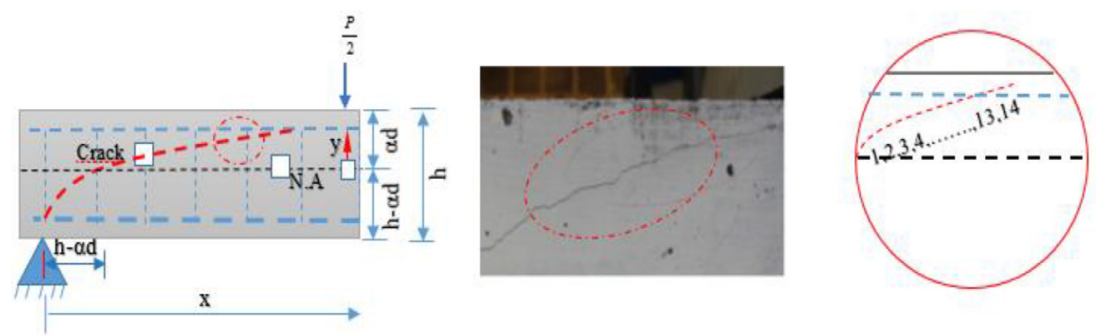

Figure 3: Numerical calculation of crack propagation into the compression zone compared with the beam tested in the laboratory.

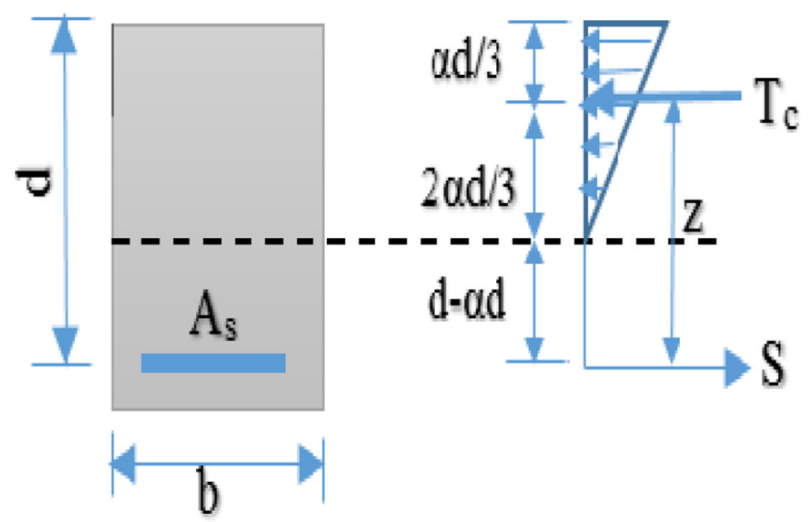

Figure 4: Internal forces in the beam.

$$
\begin{gathered}
\sigma_{x}=\frac{M_{z}(-y)}{I} \\
\sigma_{x}=\sigma_{1}=f_{c t k 0,05}
\end{gathered}
$$

Then $M=\frac{I}{(-y)} * f_{c t k 0,05}$

The height of the cross section of the beam is defined as $h$. The distance from the Neutral Axis (N.A) down to the bottom side of the beam will be $(h-\alpha . d)$ (see Fig. 4).

$$
\begin{gathered}
I_{s}=A_{s}(1-\alpha) \cdot\left(1-\frac{\alpha}{3}\right) \cdot d^{2}, z=d\left(1-\frac{1}{3} \cdot \alpha\right) \\
M_{R}=\frac{\frac{1}{2} \cdot b \cdot \alpha^{2} \cdot d^{3} \cdot\left(1-\frac{1}{3} \cdot \alpha\right)}{(-(h-\alpha \cdot d))} * f_{c t k 0,05}
\end{gathered}
$$




$$
M_{R, d}=\frac{\frac{1}{2} \cdot b \cdot \alpha^{2} \cdot d^{3} \cdot\left(1-\frac{1}{3} \cdot \alpha\right)}{(-(h-\alpha \cdot d))} * 0,567 * f_{c t k 0,05}
$$

Equation (5) describes the external moment that can be applied to the beam before it will form a crack on the underside of the beam $f_{t d}=\frac{\alpha_{c t}}{\gamma_{c}} \cdot f_{c t k 0,05}=0,567 \cdot f_{c t k 0,05}, \alpha_{c t}=0,85$ and $\gamma_{c}=1,5$ are factors given in the National Application document to EC2, [3]. Equation (6) is the design moment capacity.

\section{Modelling of Element 2:}

Vecchio \& Collins' theory of plates describes shell elements subjected to membrane shear forces. It is well known that concrete subjected to shear force cannot resist compression shear failure. This is when the compression diagonal in the truss-member-model fails.

We observe from element 2 that $\sigma_{1}$ will represent the tension force and $\sigma_{2}$ will represent compression. The combined effect of these forms a crack, see Fig. 2. We have now the basis for an inclined crack, if we consider Element 2. The shear stress can be calculated from eqn (7) as:

$$
\begin{gathered}
\tau=\frac{V}{b . z}=\sigma_{1}=f_{c t k 0,05} \\
V_{R}=b^{*} d\left(1-\frac{1}{3} * \alpha\right) * f_{c t k 0,05}
\end{gathered}
$$

The formula describes how large is the shear force that the beam can take before an inclined crack will occur at the support point of the beam $f_{t d}=\frac{\alpha_{c t}}{\gamma_{c}} \cdot f_{c t k 0,05}=0,567 \cdot f_{c t k 0,05}$ $\left(\alpha_{c t}=0,85\right.$ and $\gamma_{c}=1,5$ are factors given in the N.A EC2-NA 3.1.6, [3]).

Equation (9) is used to find the applied shear force for a concrete structure:

$$
V_{R . d}=b * d\left(1-\frac{1}{3} * \alpha\right) * 0,567 * f_{c t k 0,05}
$$

\section{Modelling of element 3:}

Element 3 is a little more complex because it varies with $\mathrm{x}$ and $\mathrm{y}$.

$$
\begin{gathered}
M_{(x)}=\frac{P}{2} \cdot x, \eta=\frac{E_{s}}{E_{c m}}, \tau_{x y}=\frac{V_{y} \cdot Q_{y}}{I_{s} \cdot b}, V_{y}=\frac{P}{2} \\
\rho=\frac{A_{s}}{b \cdot d}, \alpha=\sqrt{(\eta \cdot \rho)^{2}+2 \cdot \eta \cdot \rho}-\eta \cdot \rho \\
I_{s}=A_{s}(1-\alpha) \cdot\left(1-\frac{\alpha}{3}\right) \cdot d^{2} \\
Q_{y}=(\alpha \cdot d-y) \cdot b\left[y+\left(\frac{\alpha \cdot d-y}{2}\right)\right] \text { First moment of inertia }
\end{gathered}
$$

We insert eqns (10) and (11) 


$$
\tau_{x y}=\frac{\frac{P}{2} \cdot\left[(\alpha \cdot d-y) \cdot b\left[y+\left(\frac{\alpha \cdot d-y}{2}\right)\right]\right]}{I_{s} \cdot b}
$$

Inserting eqns (4) and (12) into eqn (1) and using the crack formation criteria $\sigma_{1}=f_{c t k 0,05}$, one can obtain the equation below. From the equation, the applied load P is solved using Mathcad, the result being given as in eqn (13):

$f_{c t k}=\frac{\frac{P}{2} \cdot x \cdot(-y)}{I_{s}}+\sqrt{\frac{\left[\frac{\frac{P}{2} \cdot x \cdot(-y)}{I_{s}}\right]^{2}}{4}+\left[\frac{\frac{P}{2} \cdot\left[(\alpha \cdot d-y] \cdot b \cdot\left[y+\left(\frac{\alpha \cdot d-y}{2}\right)\right]\right.}{I_{s} \cdot b}\right]}$ solve,

$$
\begin{gathered}
P \rightarrow\left[\begin{array}{l}
\frac{8 \cdot I_{s} \cdot f_{c t k} \cdot \sqrt{\frac{\alpha^{4} \cdot d^{4}}{4}-\frac{\alpha^{2} \cdot d^{2} \cdot y^{2}}{2}+\frac{x^{2} \cdot y^{2}}{4}+\frac{y^{4}}{4}}+4 \cdot I_{s} \cdot f_{c t k} \cdot x \cdot y}{\left(y^{2}-\alpha^{2} \cdot d^{2}\right)^{2}} \\
\frac{8 \cdot I_{s} \cdot f_{c t k} \cdot \sqrt{\frac{\alpha^{4} \cdot d^{4}}{4}-\frac{\alpha^{2} \cdot d^{2} \cdot y^{2}}{2}+\frac{x^{2} \cdot y^{2}}{4}+\frac{y^{4}}{4}}-4 \cdot I_{s} \cdot f_{c t k} \cdot x \cdot y}{\left(y^{2}-\alpha^{2} \cdot d^{2}\right)^{2}}
\end{array}\right] \\
P(x, y)=\frac{8 \cdot I_{s} \cdot f_{c t k} \cdot \sqrt{\frac{\alpha^{4} \cdot d^{4}}{4}-\frac{\alpha^{2} \cdot d^{2} \cdot y^{2}}{2}+\frac{x^{2} \cdot y^{2}}{4}+\frac{y^{4}}{4}}+4 \cdot I_{s} \cdot f_{c t k} \cdot x \cdot y}{\left(y^{2}-\alpha^{2} \cdot d^{2}\right)^{2}}
\end{gathered}
$$

The crack propagation angle is obtained by inserting eqns (2) and (12) and the result is given in eqn (14).

$$
\theta=\frac{1}{2} \tan ^{-1}\left[\frac{\left.2, \frac{\frac{P}{2} \cdot\left[(\alpha \cdot d-y) \cdot b \cdot\left[y+\left(\frac{\alpha \cdot d-y}{2}\right)\right]\right]}{I_{S} \cdot b}\right]}{\frac{\frac{P}{2} \cdot x \cdot y}{I_{S}}}\right]
$$

Equation (14) is used to determine the crack angle under various loadings, $\mathrm{P}$, in the $\mathrm{x}-\mathrm{y}$ coordinate system. $\sigma_{1}$ and $\sigma_{2}$ are dependent on the distance $\mathrm{x}$ from the support, and the distance y relative to the neutral axis. A crack will theoretically form along a critical path where the first principal stress exceeds the characteristic tensile strength of the concrete, i.e. when $\sigma_{1}=f_{c t k 0,05}$. 
The angle of the crack can be calculated considering the values of $\tau$ and $\sigma_{1}$ along this critical path.

- $x \in\left[(h-\alpha \cdot d), \frac{L}{2}\right]$

- $y \in[0, \alpha . d]$

- $y=0=>=\max , \sigma_{x}=0$

- $y=\alpha . d=>\tau=0, \sigma_{x}=\max$

\section{CRACK FORMATION IN THE COMPRESSIVE ZONE FOR ELEMENT 3}

When the concrete beam is at the stage when the concrete cracks in the compressive zone, the reinforcement is yielding. This situation leads to the needs for a non-linear stress analysis. Such analysis is beyond the scope of this paper. Our study is based on a linear stress analysis, which results in some errors for yielding reinforcement.

Using the equations derived above we can estimate the crack path as shown in Table 1 below.

$$
\begin{aligned}
& E_{S}=200000 \mathrm{MPa}, f_{y k}=500 \mathrm{MPa}, E_{\mathrm{cm}}=39000 \mathrm{MPa}, \mathrm{c}=25 \mathrm{~mm}, f_{c t k}=3,1 \mathrm{MPa} \\
& A_{S}=3 . \pi \frac{\varphi_{h}^{2}}{4} \Rightarrow A_{S}=943 \mathrm{~mm}^{2} \quad \eta=5,128 \mathrm{~mm}
\end{aligned}
$$

The following (Figs 5 and 6) illustrates how to calculate the crack propagation angle, and load for given $\mathrm{x}-\mathrm{y}$ values. The complete calculated results are shown in Table 1.

$x=h-\alpha \cdot d \Rightarrow x=218 m m \quad x_{1}=x+10 \mathrm{~mm} \cdot \cos (\theta)=>x_{1}=224.9 \mathrm{~mm}$

$y=10^{-9} \approx 0 \quad y_{1}=y+10 \mathrm{~mm} \cdot \sin (\theta)=>y_{1}=7.071 \mathrm{~mm}$

The final summary is that the method presented is innovative and it cannot be compared with results of high-tech software. It provides information and understanding why a crack is formed at high loads, and how the crack pattern develops. The intention of this paper is to show how a simple method could predict crack propagation angle based on simple beamelement theory.

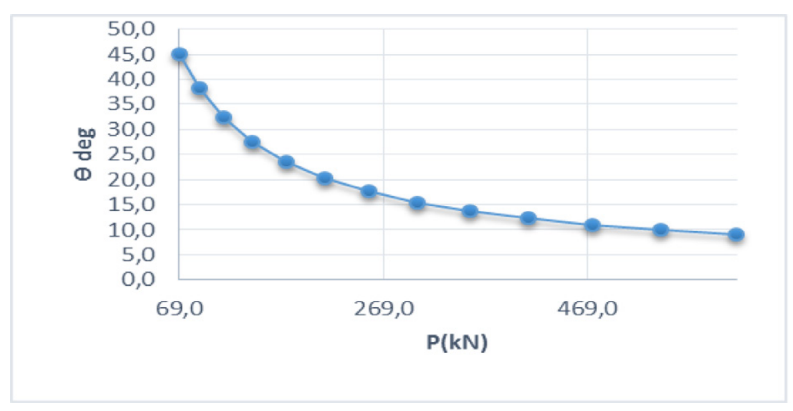

Figure 5: Effect of loading on crack propagatin angle. 


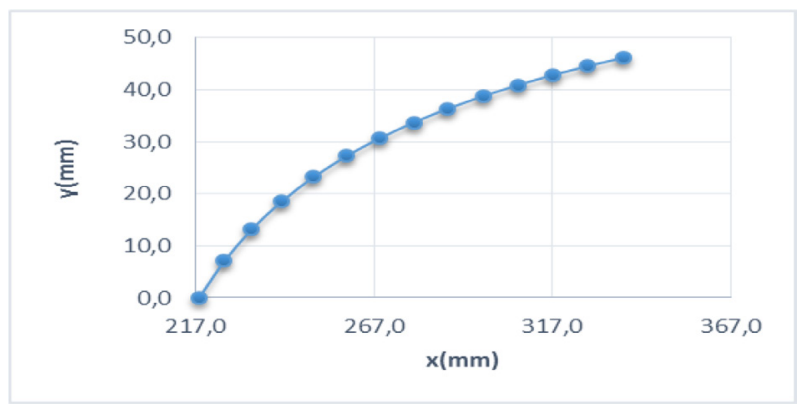

Figure 6: Crack propagatoin in $\mathrm{x}-\mathrm{y}$ axses.

Table 1: Calculation of $\mathrm{x}, \mathrm{y}, \theta$ and $\mathrm{P}$ according to Formulas given in eqns (13) and (14).

\begin{tabular}{lcccc}
\hline Crack nr. & $\mathbf{x}[\mathbf{m m}]$ & $\mathbf{y}[\mathbf{m m}]$ & $\theta[\mathbf{d e g}]$. & $\mathbf{P}[\mathbf{k N}]$ \\
\hline 1 & 217.8 & 0.0 & 45.0 & 69.4 \\
2 & 224.9 & 7.1 & 38.3 & 88.4 \\
3 & 232.7 & 13.3 & 32.4 & 112.1 \\
4 & 241.1 & 18.6 & 27.5 & 140.6 \\
13 & & & & \\
14 & 326.9 & 44.5 & 9.1 & 614.2 \\
\hline
\end{tabular}

\section{CONCLUSIONS}

The cracking of a structure reduces the performance of the structure. In the worst-case scenario, cracking may lead to failure. Furthermore, cracks to the depth of the reinforcement lead more easily to corrosion of the reinforcing bars.

The mechanism of crack formation is challenging and difficult to fully understand. However, this paper investigates the problem using mathematical and experimental methods. The method is based on a study of the crack formation when $\sigma_{1}$ exceeds the characteristics tensile strength of the concrete structure.

The commonly accepted understanding about concrete structures is that the crack formation angle is $45^{\circ}$ in the tensile zone of a supported beam. In this paper, the load dependent crack formation is experimentally and theoretically presented. Both show that as the load increases, the crack angle decreases. Finally, a proposal on how crack is propagating into the compressive zone has been presented.

\section{Notation}

As, $\quad$ Min minimum cross sectional area of reinforcement, $\mathrm{mm}^{2}$

$E_{c m} \quad$ Secant modulus of elasticity of concrete

$I_{s} \quad$ Second moment of area with respect to reinforcement

$L \quad$ Length, $\mathrm{mm}$

$Q_{y} \quad$ First moment of inertia

$S \quad$ Internal forces and moments

$T \quad$ Torsional moment

$V_{E d} \quad$ Design value of the applied shear force 
c $\quad$ Concrete cover, $\mathrm{mm}$

$d \quad$ Effective depth of a cross-section, $\mathrm{mm}$

$f_{c k} \quad$ Characteristic compressive cylinder strength of concrete at 28 days

$f_{t k} \quad$ Characteristic tensile strength of reinforcement

$f_{y k} \quad$ Characteristic yield strength of reinforcement

$x, y \quad$ Coordinate system, $x$ and $y$ variables in $x$ and $y$ directions

$z \quad$ Lever arm of internal forces, $\mathrm{mm}$

$\begin{array}{ll}a & \text { Ratio } \\ \eta & \text { Ratio } \\ \rho & \text { Reinforcement ratio for longitudinal reinforcement } \\ \phi & \text { Diameter of a reinforcing bar, } \mathrm{mm}\end{array}$

\section{REFERENCES}

[1] Beer, F.P., Russel, J. Jr, E. \& De Wolf, J.T., Mechanics of Materials, New York, 2008.

[2] Sørensen, S.I., Concrete construction, calculating and design according Eurocode 2, Trondheim, 2010.

[3] Eurocode 2: Table 8.2 of Eurocode 2: Design of concrete structures; part 1-1: General rules and rules for buildings and Norwegian Application document. Standards Norway, Oslo, 2008. 